\title{
Establishment of Reference Intervals for Serum Insulin-Like Growth Factor I in Korean Adult Population
}

\author{
Boyeon Kim ${ }^{1}$, Yonggeun Cho ${ }^{1,2}$, Cheol Ryong Kuㄹ, Sang-Guk Lee ${ }^{1}$, Kyung-A Lee ${ }^{4}$, Jeong-Ho Kim ${ }^{1}$ \\ ${ }^{1}$ Department of Laboratory Medicine, Yonsei University College of Medicine, Seoul; ${ }^{2}$ Department of Laboratory Medicine, \\ Hallym University Sacred Heart Hospital, Anyang; ${ }^{3}$ Department of Internal Medicine, Yonsei University College of Medicine, \\ Seoul; ${ }^{4}$ Department of Laboratory Medicine, Gangnam Severance Hospital, Yonsei University College of Medicine, Seoul, Korea
}

Appropriate reference intervals of serum insulin-like growth factor I (IGF-I) is important for diagnosing and monitoring patients with growth hormone-related diseases. To establish reference intervals, adult individuals ( $n=1,334,680$ men and 654 women) were divided into six age groups $(20-29,30-39,40-49,50-59,60-69, \geq 70)$. Serum IGF-I was measured by chemiluminescence immunoassay (Liaison). Concordance of patient classification based on reference intervals, manufacturer's intervals, and standard deviation score (SDS) was evaluated. New reference intervals had higher upper and lower limits than those specified by the manufacturer. The agreement between classification using new reference interval and the manufacturer's reference interval, and that using new reference interval and SDS was 75.0\% (weighted kappa, 0.17), 91.9\% (weighted kappa, 0.51) in men and 91.0\% (weighted kappa, 0.41), $92.5 \%$ (weighted kappa, 0.53 ) in women, respectively. Reference intervals should be established not only based on age and sex, but also on ethnicity and assay method.

Keywords: Insulin-like growth factor I; Reference values; Luminescent measurements

\section{INTRODUCTION}

The level of insulin-like growth factor I (IGF-I) is affected by various factors such as age, sex, systemic illness, ethnicity, and the type of the analytical method $[1,2]$. Therefore, reference intervals of IGF-I considering those factors are necessary for the interpretation of measurement values in a given population.

A few studies have been conducted among Asian populations. Hyun et al. [3] and Choi et al. [4] measured IGF-I and insulinlike growth factor-binding protein 3 (IGFBP-3) levels using immunoradiometric assays in Korean population; however, this assay is not commonly used owing to the generation of radioisotope waste. Zhu et al. [5] recently published reference inter- vals for IGF-I in healthy Chinese adults using a chemiluminescence immunoassay (Immulite 2000, Siemens Healthcare Diagnostics, Gwynedd, UK). However, this study enrolled insufficient numbers of subjects in each group to set the reference intervals [6]. Therefore, it is necessary to establish reference intervals for IGF-I based on assay methods by including a sufficient number of subjects in each age group, especially for the Asian population.

Here, we measured IGF-I levels using a chemiluminescence immunoassay (Liaison XL, DiaSorin S.p.A., Saluggia, Italy) and established reference intervals to improve the accuracy of diagnosis and treatment monitoring.
Received: 10 June 2020, Revised: 18 September 2020,

Accepted: 30 September 2020

Corresponding author: Sang-Guk Lee

Department of Laboratory Medicine, Severance Hospital, Yonsei University College of Medicine, 50-1 Yonsei-ro, Seodaemun-gu, Seoul 03722, Korea Tel: +82-2-2228-2455, Fax: +82-2-364-1583, E-mail: comforter6@yuhs.ac
Copyright $\odot 2020$ Korean Endocrine Society

This is an Open Access article distributed under the terms of the Creative Commons Attribution Non-Commercial License (https://creativecommons.org/ licenses/by-nc/4.0/) which permits unrestricted non-commercial use, distribution, and reproduction in any medium, provided the original work is properly cited. 


\section{METHODS}

Information regarding 5,010 patients (2,079 men, 2,931 women) who visited outpatient clinics (Plastic Surgery, Orthopedics, Ophthalmology, Dermatology, Family Medicine, Dentistry, Otorhinolaryngology, Allergy and Immunology) from December 2018 to July 2019 was reviewed, and excluded based on the following exclusion criteria: (1) a medical history of liver diseases, renal diseases, cancer, diabetes mellitus, cardiac disorder, pulmonary disorder, or thyroid disorder or alanine aminotransferase $>54$ $\mathrm{IU} / \mathrm{L}$, aspartate aminotransferase $>32 \mathrm{IU} / \mathrm{L}$, estimated glomerular filtration rate $<60 \mathrm{~mL} / \mathrm{min} / 1.73 \mathrm{~m}^{2}$, glucose $\geq 126 \mathrm{mg} / \mathrm{dL}$, or C-reactive protein $>10 \mathrm{mg} / \mathrm{L}$; (2) no test results for liver enzyme, glucose, and creatinine; (3) the use of estrogen medication; and (4) ethnicity other than Korean. We defined "healthy" subjects to be those who passed all the exclusion criteria. After exclusion, a total of 1,358 subjects ( 688 men and 670 women) were selected. These participants were divided into six age groups (20-29, 30-39, 40-49, 50-59, 60-69, and $\geq 70$ years). IGF-I was measured by Liaison XL. This study was approved by the Institutional Review Board of Severance Hospital (IRB No. 4-2018-1065). Written informed consent by the patients was waived due to a retrospective nature of our study.

After measuring IGF-I, Tukey method to remove outliers (eight men and 16 women) [7] and Anderson-Darling test to analyze the normality were applied. The skewed distributions of IGF-I in men aged 30 to 39 and 40 to 49 years were normalized using logarithmic transformation. Then we established the reference intervals using a parametric method, known to be most powerful when a random sample is obtained from a normally distributed population [8].

IGF-I value from each subject was classified into low, normal, and high based on the following: (1) reference intervals provided by the manufacturer; (2) those obtained in a previous study to allow standard deviation score (SDS) calculation; and (3) those derived in this study. To obtain the SDS, we used a calculator available online (http://ticemed_sa.upmc.fr/sd_score/) [9]. The categories are as followings: high (SDS >1.96), normal (SDS between -1.96 and 1.96), and low (SDS $<-1.96$ ). The agreement of classification among three reference intervals, expressed as weighted kappa and percentage of observed agreement, was evaluated.

All statistical analyses were performed using Microsoft Excel 2010 (Microsoft Corporation, Redmond, WA, USA), Analyse-it for Microsoft Excel Method Evaluation Edition version e.76.1 (Analyse-it Software Ltd., Leeds, UK), and psych package of R software version 3.6.1 (R Core Team, 2019; R: A language and environment for statistical computing; R Foundation for Statistical Computing; http://www.r-project.org). To interpret the kappa values, Landis and Koch's Table was used [10].

\begin{tabular}{|c|c|c|c|c|c|}
\hline \multirow{2}{*}{ Age group, yr } & \multirow{2}{*}{ No. } & \multirow{2}{*}{ Mean } & \multirow{2}{*}{ SD } & \multicolumn{2}{|c|}{ Reference intervals for IGF-I, ng/mL } \\
\hline & & & & Lower limit $(90 \% \mathrm{CI})$ & Upper limit (90\% CI) \\
\hline \multicolumn{6}{|l|}{ Men } \\
\hline $20-29$ & 147 & 262.79 & 68.09 & $127.77(111.98-143.55)$ & $397.81(382.03-413.60)$ \\
\hline $30-39$ & 130 & 224.36 & 54.73 & $132.13(124.19-140.57)$ & $358.53(336.99-381.44)$ \\
\hline $40-49$ & 124 & 199.15 & 52.78 & $110.09(102.58-118.14)$ & $334.69(311.87-359.18)$ \\
\hline $50-59$ & 137 & 185.94 & 55.20 & $76.38(63.12-89.64)$ & $295.50(282.24-308.76)$ \\
\hline $60-69$ & 78 & 166.06 & 47.82 & $72.03(56.81-87.25)$ & $260.09(244.87-275.31)$ \\
\hline$\geq 70$ & 64 & 137.33 & 41.74 & $55.19(40.52-69.86)$ & $219.47(204.80-234.13)$ \\
\hline \multicolumn{6}{|l|}{ Women } \\
\hline $20-29$ & 124 & 256.99 & 71.70 & $114.50(96.40-132.60)$ & $399.49(381.39-417.59)$ \\
\hline $30-39$ & 119 & 222.41 & 53.37 & $116.28(102.52-130.03)$ & $328.54(314.79-342.30)$ \\
\hline $40-49$ & 128 & 192.67 & 48.54 & $96.25(84.19-108.31)$ & $289.09(277.03-301.15)$ \\
\hline $50-59$ & 135 & 160.19 & 48.49 & $63.94(52.21-75.67)$ & $256.45(244.72-268.18)$ \\
\hline $60-69$ & 85 & 144.88 & 47.84 & $50.83(36.24-65.42)$ & $238.93(224.35-253.52)$ \\
\hline$\geq 70$ & 63 & 117.57 & 44.98 & $29.05(13.12-44.98)$ & $206.09(190.16-222.02)$ \\
\hline
\end{tabular}

IGF-I, insulin-like growth factor I; SD, standard deviation; CI, confidence interval. 


\section{RESULTS}

After excluding outliers, the final reference population comprised 1,334 subjects ( 680 men and 654 women). There were 147, 130, $124,137,78$, and 64 men and 124, 119, 128, 135, 85, and 63 women in the $20-29,30-39,40-49,50-59,60-69$, and $\geq 70$ years age groups, respectively.

In every age group, lower mean concentration in women than in men is observed; however, it is statistically significant only after the age of 50, the postmenopausal states. The IGF-I reference intervals (Table 1) showed that the mean values and upper limits of reference intervals decreased with an increase in age, while the lower limit showed a similar trend except for the age groups 20 to 29 and 30 to 39 (in both men and women). The new reference intervals were significantly higher in the upper limit especially in the age groups below 60 years compared to the Liaison package insert (Fig. 1), and the difference was much higher in men than in women.

IGF-I values were classified as low, normal, and high according to the new reference intervals, package insert, and SDS. Based on the classification, the agreement between new IGF-I
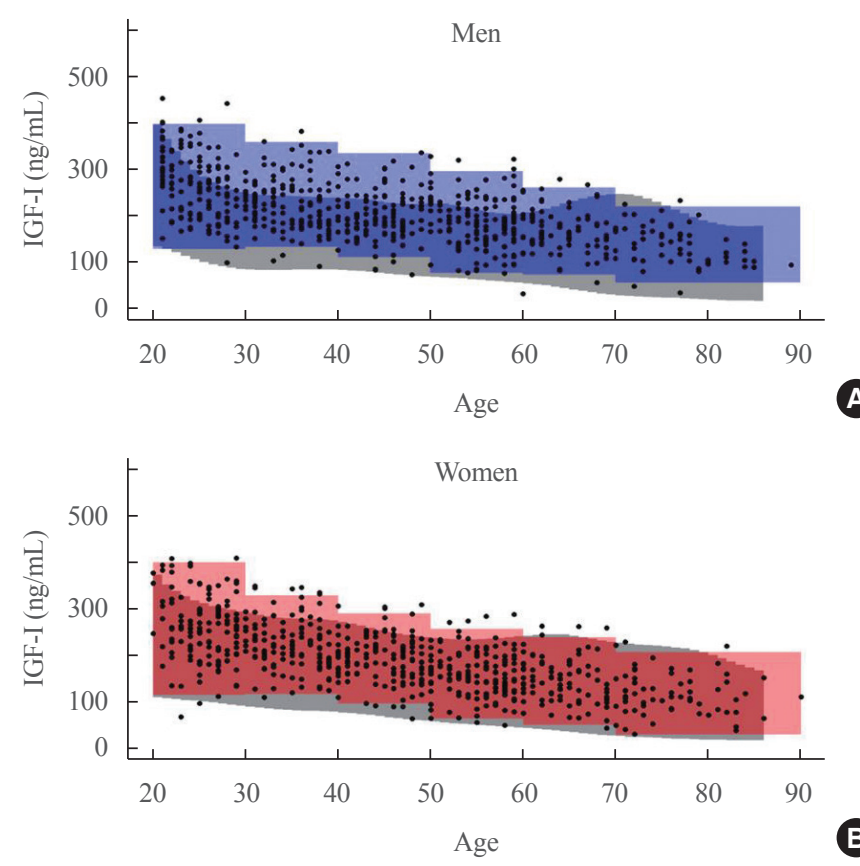

Fig. 1. Individual data and comparison between the new reference intervals and reference intervals of Liaison insert. The black points indicate individual data. New reference intervals are shown in colored section (A: blue, men; B: red, women). Reference intervals of Liaison insert paper are shown in the grey section. Liaison insert paper only showed reference intervals until the age of 85 years. IGF-I, insulin-like growth factor I. reference intervals and manufacturer's reference intervals was low and moderate with a kappa coefficient of 0.17 and 0.41 in men and women, respectively. Further, the agreement between new IGF-I reference intervals and SDS was moderate with a kappa coefficient of 0.51 and 0.53 in men and women, respectively (Supplemental Table S1).

\section{DISCUSSION}

Ethnicity and the type of assay method employed are important when measuring and interpreting the IGF-I levels. Compared to the mean concentration levels of IGF-I in this study, lower values were reported when measured with the immunoradiometric method except for the women aged below 30 (Supplemental Table S2) [4]. To overcome the interlaboratory variability in patient classification, SDS calculator was established [1,9]; however, we observed a moderate agreement between the new reference intervals and SDS in men (kappa, 0.51) and women (kappa, 0.53 ). This unsatisfactory agreement could be related to ethnicity, as the reference intervals provided by the manufacturer and SDS calculator were established in Europe. Therefore, the new reference intervals derived herein may be used in hospitals using Liaison, especially while interpreting IGF-I values in Asian adults. Despite the moderate concordance with SDS, the SDS calculator could be helpful to get an idea regarding patient classification; however, as racial differences are expected, an additional multicenter study comparing IGF-I reference intervals in Asian healthy population should be conducted.

The present study has several limitations. First, despite of strictly designed exclusion criteria for selecting patients with most likely not affecting the growth hormone/IGF-I system, the definition of "healthy" population in this study may not absolutely reflect the consensus requirement of a well characterized cohort of healthy subjects [11]. For example, we might have not excluded all the subjects with possible endocrine disorder or chrnoic infective diseases. Second, it was difficult to collect the data about hypertension, or pregnancy or menopausal status, or time of blood sample was taken due to the retrospective study design. Generally, the patients who visited outpatient clinics in our institute were asked to have a blood testing after having desirably overnight or at least 4 hours of fasting. Third, although the analysis of fasting glucose level between patients classified into the same IGF-1 group and those classified into different group showed no significant difference, we could not completely confirm that nutritional condition had no effect on the discordant result due to lack of body mass index information. Fourth, 
although we excluded the subjects using estrogens, we could not have information for the use of other steroids $[12,13]$. Finally, this study was conducted in one center in urban area, therefore multicenter study to confirm the reference intervals of IGFI should be needed.

Despite these limitations, it should be noted that we enrolled at least 120 apparently healthy subjects based on strict exclusion criteria in group except the age over 60 years. As the kidney and liver functions and diabetic status may affect the level of IGF-I $[14,15]$, we thoroughly examined patient medical history, liver profile, creatinine, and glucose levels. To our knowledge, this is the first study to establish IGF-I reference intervals measured with a chemiluminescence method in Korean population using an adequate number of reference subjects.

In conclusion, we have established reference intervals for IGF-I measured by Liaison XL. As various factors affect the levels of IGF-I, reference intervals should be established based on not only age and sex but also on ethnicity and the assay method employed.

\section{CONFLICTS OF INTEREST}

No potential conflict of interest relevant to this article was reported.

\section{ACKNOWLEDGMENTS}

This study was supported by Basic Science Research Program through the National Research Foundation of Korea (NRF) funded by the Ministry of Science, ICT and Future Planning (NRF-2020R1F1A1051360).

\section{AUTHOR CONTRIBUTIONS}

Conception or design: B.K., S.G.L., J.H.K. Acquisition, analysis, or interpretation of data: B.K., Y.C., S.G.L., J.H.K. Drafting the work or revising: B.K., C.R.K., S.G.L., K.A.L., J.H.K. Final approval of the manuscript: B.K., Y.C., C.R.K., S.G.L., K.A.L., J.H.K.

\section{ORCID}

Boyeon Kim https://orcid.org/0000-0003-1867-8648

Sang-Guk Lee https://orcid.org/0000-0003-3862-3660

\section{REFERENCES}

1. Mavromati M, Kuhn E, Agostini H, Brailly-Tabard S, Massart C, Piketty ML, et al. Classification of patients with GH disorders may vary according to the IGF-I assay. J Clin Endocrinol Metab 2017;102:2844-52.

2. Dogansen SC, Yalin GY, Tanrikulu S, Yarman S. Impact of glucose metabolism disorders on IGF-1 levels in patients with acromegaly. Horm Metab Res 2018;50:408-13.

3. Hyun SE, Lee BC, Suh BK, Chung SC, Ko CW, Kim HS, et al. Reference values for serum levels of insulin-like growth factor-I and insulin-like growth factor binding protein-3 in Korean children and adolescents. Clin Biochem 2012;45: 16-21.

4. Choi HK, Kong MH, Ahn BH, Kim SM, Lee DJ, Kim KM. Insulin-like growth factor 1 level and its relating factor in Korean healthy adults. Korean J Fam Med 2009;30:15-22.

5. Zhu H, Xu Y, Gong F, Shan G, Yang H, Xu K, et al. Reference ranges for serum insulin-like growth factor I (IGF-I) in healthy Chinese adults. PLoS One 2017;12:e0185561.

6. Clinical and Laboratory Standards Institute. Defining, establishing, and verifying reference intervals in the clinical laboratory; approved guideline. 3rd ed. Wayne: Clinical and Laboratory Standards Institute; 2008.

7. Tukey JW. Exploratory data analysis. Reading: Addison-Wesley Pub; 1977.

8. Solberg HE. Approved recommendation (1987) on the theory of reference values. Part 5. Statistical treatment of collected reference values. Determination of reference limits. Clin Chim Acta 1987;170:S13-32.

9. Chanson P, Arnoux A, Mavromati M, Brailly-Tabard S, Massart C, Young J, et al. Reference values for IGF-I serum concentrations: comparison of six immunoassays. J Clin Endocrinol Metab 2016;101:3450-8.

10. Landis JR, Koch GG. The measurement of observer agreement for categorical data. Biometrics 1977;33:159-74.

11. Clemmons DR. Consensus statement on the standardization and evaluation of growth hormone and insulin-like growth factor assays. Clin Chem 2011;57:555-9.

12. Gayan-Ramirez G, Vanderhoydonc F, Verhoeven G, Decramer M. Acute treatment with corticosteroids decreases IGF1 and IGF-2 expression in the rat diaphragm and gastrocnemius. Am J Respir Crit Care Med 1999;159:283-9.

13. Prummel MF, Wiersinga WM, Oosting H, Endert E. The effect of long-term prednisone treatment on growth hormone and insulin-like growth factor-1. J Endocrinol Invest 1996; 
19:620-3.

14. Clemmons DR. Value of insulin-like growth factor system markers in the assessment of growth hormone status. Endo- crinol Metab Clin North Am 2007;36:109-29.

15. Teppala S, Shankar A. Association between serum IGF-1 and diabetes among U.S. adults. Diabetes Care 2010;33:2257-9. 